\title{
An M/M/1 Preemptive Queue based Priority MAC Protocol for WBSN to Transmit Pilgrims' Data
}

\author{
Shah Murtaza Rashid Al Masud ${ }^{1}$, Mahmood ul Hassan², Khalid Mahmood ${ }^{3}$, Muhammad Akram ${ }^{4}$ \\ Department of Computer Science, IIC University of Technology, Phnom Penh, Kingdom of Cambodia ${ }^{1,2}$ \\ Department of Computer Skills, Najran University, Najran, Kingdom of Saudi Arabia ${ }^{2}$ \\ Department of Information Systems, Tehama Branch, King Khalid University, Abha, Kingdom of Saudi Arabia ${ }^{3}$ \\ College of Computer Science and Information systems, Najran University, Najran, Kingdom of Saudi Arabia $a^{1,4}$
}

\begin{abstract}
Every year during Hajj in Saudi Arabia and Kumbh Mela in India, many pilgrims suffering from different medical emergencies thus need real-time and fast healthcare services. Quick healthcare can be facilitated by setting up Wireless Body Sensor Network (WBSN) on pilgrims because of its suitability for a wide range of medical applications. However, higher delay, data loss and excessive energy consumption may occur in the network when multiple emergency data aggregate at the coordinator for accessing the data communication channel simultaneously. In this context, for low delay and energy-efficient data transmission, an $M / M / 1$ preemptive queue technique is proposed and minimal backoff period is considered to develop a priority Medium Access Control (MAC) protocol for WBSN. Our proposed MAC is designed based on IEEE802.15.6 standard that supports modified MAC superframe structure for heterogeneous traffic. The proposed priority MAC protocol has been simulated using the Castalia simulator to analyze the results. In the first scenario considering varying nodes, the delay is calculated as $13 \mathrm{~ms}$ and $33 \mathrm{~ms}$ for the emergency, and the normal medical condition. Besides, for emergency and normal medical condition energy consumption per bit is calculated at around $0.12 \mu \mathrm{j}$ and $0.19 \mu \mathrm{j}$. In the second scenario, we consider variation in traffic size. For 16 bytes traffic size, delay of extremely very high critical traffic is $5.8 \mathrm{~ms}$ and $14.5 \mathrm{~ms}$ for extremely low critical traffic. Similarly, extremely very high critical traffic consumes $0.035 \mu \mathrm{j}$ energy per bit, whereas extremely low critical traffic consumes $0.37 \mu \mathrm{j}$. in the third scenario, the delay, data loss rate, average energy consumption and throughput for the proposed priority MAC are analyzed. Result demonstrates our proposed priority MAC protocol outperforms the state-of-the-art protocols.
\end{abstract}

Keywords-Wireless body sensor network; medium access control protocol; preemptive queue; priority; heterogeneous traffic

\section{INTRODUCTION}

According to the World Health Organization (WHO), the number of pilgrims from all over the world congregates to perform Hajj is more than two million. During Kumbh Mela in India, around sixty million pligims congregate at different four cities. Among many problems during the pilgrimage, pilgrims health monitoring is the most crucial issue. During the expedition, it is essential to identify people with health risk. Once they are detected, it is easy to monitor them and medical help and assistant can easily be provided for them [1-5].
So far, a few tracking and monitoring systems are proposed including, GPS as trackers [6], pilgrim's locator system [7], Hajj Locator for tacking pilgrims in a crowded environment [8]. RFID based location-tracking system [9]; WSNs based intelligent transportation systems (ITS) for facilitating efficient traffic movements [10]. Therefore, the healthcare sectors during Hajj and other religious festivals are looking for advanced Information and Communication Technology (ICT), which will be able to give health care services to pilgrims' in a wide-crowded area. There is a massive demand for deploying a new technology that can monitor and provide a real-time treatment to the pilgrims throughout the whole pilgrimage routine.

Wireless Body Sensor Networks (WBSNs) are offering a quick evolution in patients' healthcare monitoring applications. There are plenty of possibilities where location independent WBSNs are useful for monitoring, diagnosis, and treatment of diseases [11]. The IEEE 802.15.6 standard based WBSN/WBAN facilitates to deal with heterogeneous traffic include emergency and routine medical traffic. However, emergency data is mandatory to transmit ahead of other nonemergency traffic because any delay or data lost during transmission may impede patients' life [11].

Emergency traffic is very unpredictable and may produce regularly and eve randomly. Generally, emergency data are to be transmitted in non-scheduled and contention-free phase. However, normal (regular) medical data are to be sent in contention access period. But, the problem occurs when multiple emergency data aggregate at body coordinator to be transmitted simultaneously in WBAN medium thus results in data collision, data loss, severe delay and energy inefficiency. Hence, for collision-free, low delay and power-efficient data transmission, it is significant to identify the criticality level of emergency traffic to set the priority of emergency traffic. Hence, in this research, we proposed an $\mathrm{M} / \mathrm{M} / 1$ preemptive queue-based priority MAC protocol to efficiently transmit pilgrims' data to the healthcare station with no delay and an energy-efficient manner. In a preemptive priority queue approach, data with high priority should access the communication channel faster and ahead of low priority data. Besides, for energy-efficient and low delay data transmission, a modified MAC superframe is proposed. Where Exclusive Access Phase (EAP) is fixed for emergency data transmission and Managed Access Phase is allocated for normal medical data transmission in WBSN medium. 
The proposed priority MAC protocol has been simulated using Castalia simulator [20] to analyze and validate the results. The performance metrics of the proposed MAC protocol are analyzed and verified with state-of-the-art protocols. Results are compared with the most recent traffic adaptive based MAC protocol (TA-MAC) and traffic priority based channel access technique (TPCAT) protocol. Results demonstrate that delay, data loss rate, and the average energy consumption are relatively low, and the throughput is high during emergency data transmission in a network due to no data retransmission and collision. The main drawback of this research is to consider limited quality of service (QoS) requirement and limited biomedical data for our proposed priority MAC protocol.

The remainder of the paper is organized as follows: In Section 2, the related work is introduced. WBSN traffic classification, prioritization, network management are presented in Section 3. Section 4 shows a discussion for the simulation results. Finally, the paper ends with a conclusion in Section 5.

\section{RELATED WORK}

This section reviews the literature to analyze different methods, mechanisms, schemes that are available and applied for developing priority MAC protocol for medical applications. The priority MAC protocol must consider heterogeneous traffics. It should deem about different application requirements and the key challenges, including high data rates and prioritization of critical emergency data, low energy consumption, and low delay. The MAC protocol must ensure the timely delivery of medical data in the WBSN medium because any loss of such critical data may jeopardize the life of patients.

For better traffics adaptation and higher energy efficiency, the IEEE802.15.6 standard-based adaptive MAC protocol with modified superframe (A-MAC) is proposed [12]. A novel energy-efficient and lower delay MAC protocol for WBAN by using the radio wake-up mechanism is proposed [13]. IEEE802.15.4 standard-based traffic-aware and reliable MAC for WBAN is offered by [14]. To design the protocol, the authors classified the emergency traffics into different criticality levels based on the threshold value. The superframe is modified and divided into different time slots for channel access. A dynamically allocated time slot for IEEE802.15.4 based MAC superframe is proposed by the authors [15], CAP is divided into four phases. The data is prioritized into four different types, and according to the level of priority data get access to the phases as emergency traffic ET-CAP (Phase 1), on-demand traffic ODT CAP (Phase 2), normal traffic NTCAP (Phase 3), and non-medical traffic NMT-CAP (Phase 4).

A traffic priority based channel assignment technique (TPCAT) for critical traffic is proposed by [16], which is based on IEEE802.15.6. The method is developed based on two algorithms include low threshold and high threshold adaptive time slot algorithms. The main goal of TP-CAT is set to low delay data transmission for critical data. Traffic-adaptive priority MAC protocol (TAP-MAC) is proposed [17] using a modified MAC superframe structure aims to reduce contention in the CAP period; otherwise, the collisions in data transmission channels may increase. Energy-Efficient Traffic Prioritization scheme (EETP) with modified MAC superframe is proposed in [18]. Where traffic is prioritized into four categories according to delay, reliability and criticality constraints [18], besides, both CSMA/CA and TDMA techniques are used to access the channel for non-emergency and life-critical emergency traffic, respectively.

Traffic sensitive WBAN by using TDMA based nonpreemptive priority queue method has been proposed by [19]. Three different traffic priority levels, including emergency traffic, on-demand and normal traffic, are being classified and non-preemptive queues method at the WBAN coordinator has been proposed for data transmission in the medium. In this study, similar types of data need to wait for transmitting in the pre-reallocated time slots; as a result, delay and energy consumption both are increased.

Most of the existing protocols, as explained above, are proposed based on the IEEE 802.15.4 and the IEEE 802.15.6 standards that pay attention to a particular class of traffic either high or low. In WBAN, high-priority traffic is usually the lowest load traffic. And low-priority traffic is usually the highest load traffic, hence ignoring both or one of them the MAC superframe may strictly harm the overall transmission, energy consumption, overall delay and network throughput. Different data types reflect the primary motivation of almost all the MAC protocol mentioned above. However, there is a crucial necessity to provide additional importance to handle various emergency data and its criticality levels. The observation on the shortcomings of the current WBSN priority and traffic management protocol has encouraged us to develop a novel traffics' management solution for a network to cope with challenges above including lower delay and lower energy consumption; and higher network throughput.

\section{WBSN OPERATION FOR THE PROPOSED PRIORITY MAC PROTOCOL}

\section{A. Data Classification and Prioritization}

To deal with a normal (regular) medical and emergency event at any moment is much more comfortable, encouraging and less challenging than that of multiple emergency events simultaneously at WBSN coordinator level. Based on our study [1-7], to build a priority MAC protocol in this research, we consider heterogeneous traffic. Besides, emergency data is further classified into twelve different classes based on traffics severity level during the pilgrimage as presented in the following Table I.

Emergency critical data are usually event-triggered traffic and is produced whenever a life-threatening circumstance occurs. Hence, it is to be delivered and transmitted in WBAN medium with no delay, no loss and in a timely and real-time manner. On the other hand, non-critical normal physiological data require periodic monitoring hence does not restrict to strict delay or reliability constraints. 


\section{B. Proposed MAC Superframe Structure}

The entire communication channel of the IEEE 802.15.6 standard is divided into superframe structures. Each superframe is encircled by a beacon period of equal length. To avoid data collision and simplicity of the research work, we further modify the MAC superframe, which is presented in Fig. 1.

In our proposal, we assign an Exclusive Access Phase (EAP) for emergency traffic that is a combined version of both EAP1 and EAP2. User priority $\mathrm{UP}_{7}$ is set to ensure its highest level of priority over all sorts of medical data. Emergency medical data are aperiodic and required contention-free access to the channel, which is not scheduled based. Besides, in our proposed mechanism, for on-demand medical data Random Access Period (RAP) is proposed that is a combined version RAP1 and RAP2. And finally, for normal medical or nonmedical traffic a scheduled based or query-based Managed Access Period or phase (MAP) is proposed, which is a combined version of both MAP 1 and MAP 2. It can be observed from the above discussion that, in this research, sensor nodes use a priority-aware CSMA/CA access scheme for EAP access phases along with preemptive priority queue model to attain contention-free data communication and nodes allocation.

TABLE I. SEVERITY LEVEL OF DiSEASES

\begin{tabular}{|l|l|}
\hline Disease & Severity level \\
\hline $\begin{array}{l}\text { Respiratory diseases such as } \\
\text { pneumonia, influenza, asthma, }\end{array}$ & extremely very high critical traffic \\
\hline $\begin{array}{l}\text { Heart attack due to heatstroke or heat } \\
\text { attack }\end{array}$ & Extremely high critical traffic \\
\hline Diabetes due to blood sugar & Extremely critical traffic \\
\hline $\begin{array}{l}\text { Cardiovascular disease (Heart } \\
\text { disease) }\end{array}$ & Very high critical traffic \\
\hline Blood pressure & High critical traffic \\
\hline Gastroenteritis & Critical traffic \\
\hline Hypertension & Moderately critical traffic \\
\hline Skin disease, Dry eye & Moderately low critical traffic \\
\hline $\begin{array}{l}\text { High Fever or High Body } \\
\text { Temperature }\end{array}$ & Moderately very low critical traffic \\
\hline Urinary tract infection (UTI) & Low critical traffic \\
\hline Diarrhoea and jaundice & Very low critical traffic \\
\hline Meningococcal & Extremely low critical traffic \\
\hline
\end{tabular}

\begin{tabular}{|c|c|c|c|c|}
\hline Beacon & $\begin{array}{c}\text { UP7 } \\
\text { CSMA/CA }\end{array}$ & $\begin{array}{c}\text { All UPs } \\
\text { CSMA/CA }\end{array}$ & $\begin{array}{c}\text { Polling } \\
\text { Mechanism }\end{array}$ & Beacon \\
\hline \multirow[t]{2}{*}{ B } & EAP & RAP & MAP & \multirow[t]{2}{*}{ B } \\
\hline & $\begin{array}{l}\mathrm{T}_{\text {EAP }} \text { belong to } \\
\text { Emergency } \\
\text { medical data }\end{array}$ & $\begin{array}{l}T_{R A p} \text { belong to } \\
\text { On-demand data }\end{array}$ & $\begin{array}{l}\mathrm{T}_{\text {MAP }} \text { belong to } \\
\text { Normal medical } \\
\text { data }\end{array}$ & \\
\hline \multicolumn{5}{|c|}{ Beacon Period (Superframe) } \\
\hline $\begin{array}{l}\text { EAP: ex } \\
\text { RAP: rar } \\
\text { MAP: } m\end{array}$ & $\begin{array}{l}\text { lusive access ph } \\
\text { dom access pha } \\
\text { naged access pl }\end{array}$ & & & \\
\hline
\end{tabular}

Fig. 1. Proposed MAC Superframe Structure.
In this research, we suppose, emergency data, on-demand data and normal data comprise the highest priority, the medium priority, and the lowest priority respectfully. Also, for collecting emergency data considering different criticality levels, five sensors are to be utilized, followed by one sensor for on-demand data. Normal physiological data are to be collected periodically by the same sensors that are deployed for emergency traffic. Usually, emergency or critical data and normal physiological data can easily be differentiated by their threshold values. Thus, the priority-criticality index for the data is defined by the combination of the highest level of user priority $\left(\mathrm{P}_{1}\right.$ : emergency data) and different data criticality level $\left(\mathrm{C}_{1}-\mathrm{C}_{12}\right)$. The index (I) is initiated to identify the criticality stage of emergency data as $I=P_{1} C_{i}$ where $i=1-12$, that ranges from $\mathrm{P}_{1} \mathrm{C}_{1}-\mathrm{P}_{1} \mathrm{C}_{12}$ with WBAN user priority 7. Moreover, the higher the index, the higher the emergency data criticality level. Therefore the emergency traffic with the highest criticality level gets privileged to communicate faster in WBAN medium followed by on-demand traffic $\left(\mathrm{P}_{2}\right)$ with WBAN user priority 6 , and normal traffic $\left(\mathrm{P}_{3}\right)$ with WBAN user priority 5 . Body sensors and body coordinator have different activities such as sensors sense, process and transmit data to coordinator; whereas, coordinator collects, processes, sorts, and transmits data for further processing at healthcare centres. The following Table II illustrates the severity (criticality)-priority index table.

\section{The Functions of Sensor Nodes and Coordinator}

To deal with heterogeneous traffic for the proposed priority MAC protocol, we deploy an $\mathrm{M} / \mathrm{M} / 1$ preemptive priority queue with different user priority and other classes of traffics. An M/M/1 queue deals with one server and one channel (wireless medium) where both the inter-arrival time and service time are exponentially distributed and arrivals are determined by the Poisson process. Besides, the service time (Backoff process duration) of the $\mathrm{M} / \mathrm{M} / 1$ priority queue model does not follow any universal probability law and the Poisson process can model the generated traffic in the system. In this proposed model, emergency traffic is classified and prioritized through the severity level of pilgrims' health-related problems. From the numerical analysis, we find that the physiological data with the uppermost priority should not stay at the queue for a long time which reduces the overall delay during communication. Furthermore, for energy-efficient data transmission, we suggest a sleep/idle-wakeup method that decreases unnecessary energy consumption.

The sensor node sensed data and send it to the coordinator node. After receiving data from sensors, the coordinator node compares the sensed data with the pre-defined threshold value to define whether the event is an emergency or not. An emergency event occurs when data exceeds the pre-defined threshold value. Otherwise, data belong to either on-demand (aperiodic) or normal (periodic). The data transmission rate is higher in emergency condition than on-demand or normal state. Once data types are defined, data are sent to the nearest access point through the data communication channel. Emergency data is further classified and send to healthcare stations through dedicated time slot based on the criticality level. 
TABLE II. SEVERITY (CRITICALITY)-PRIORITY INDEX TABLE

\begin{tabular}{|c|c|c|c|c|}
\hline $\begin{array}{l}\text { Providing } \\
\text { User Priority } \\
\text { (UP) } \\
\text { according to } \\
\text { IEEE802.15. } \\
6 \text { standard }\end{array}$ & $\begin{array}{l}\text { Index of } \\
\text { Data } \\
\text { Priority }\end{array}$ & $\begin{array}{l}\text { Index of } \\
\text { Emergency Data } \\
\text { Criticality }\end{array}$ & $\begin{array}{l}\text { Criticality- } \\
\text { Priority } \\
\text { Index } \\
\text { Table }\end{array}$ & $\begin{array}{l}\text { Types of } \\
\text { Access } \\
\text { Phases }\end{array}$ \\
\hline $\begin{array}{l}\mathrm{UP}_{7} \text { for the } \\
\text { Emergency } \\
\text { medical } \\
\text { situation }\end{array}$ & $\begin{array}{l}\mathrm{P}_{1}, \\
\text { indicate } \\
\mathrm{s} \text { the } \\
\text { highest } \\
\text { priority }\end{array}$ & $\begin{array}{l}C_{i}=\{1,2,3,4,5, \ldots, 12 \\
\}\end{array}$ & $\begin{array}{l}\text { Indices for } \\
\text { different } \\
\text { emergency } \\
\text { data: } \\
\mathrm{P}_{1} \mathrm{C}_{\mathrm{i}}=\left\{\mathrm{P}_{1} \mathrm{C}_{1}\right. \\
\mathrm{P}_{1} \mathrm{C}_{2}, \mathrm{P}_{1} \mathrm{C}_{3}, \\
\mathrm{P}_{1} \mathrm{C}_{4}, \mathrm{P}_{1} \mathrm{C}_{5}, \\
\left.\ldots \ldots, \mathrm{P}_{1} \mathrm{C}_{12}\right\}\end{array}$ & $\begin{array}{l}\text { EAP } \\
\text { (EAP1 } \\
\text { and } \\
\text { EAP2)- } \\
\text { Exclusiv } \\
\text { e Access } \\
\text { Phase }\end{array}$ \\
\hline $\begin{array}{l}\mathrm{UP}_{6} \text { for high } \\
\text { priority } \\
\text { medical data }\end{array}$ & $\begin{array}{l}\mathrm{P}_{2}, \\
\text { indicate } \\
\mathrm{s} \text { the } \\
\text { medium } \\
\text { priority }\end{array}$ & $\mathrm{P}_{1} \mathrm{C}_{\mathrm{i}}=0$ & & $\begin{array}{l}\text { RAP } \\
\text { (RAP1 } \\
\text { and } \\
\text { RAP2)- } \\
\text { Random } \\
\text { Access } \\
\text { Phase } \\
\end{array}$ \\
\hline $\begin{array}{l}\mathrm{UP}_{5} \text { for } \\
\text { medical data }\end{array}$ & $\begin{array}{l}\mathrm{P}_{3} \text {, } \\
\text { indicate } \\
\mathrm{s} \text { the } \\
\text { lowest } \\
\text { priority }\end{array}$ & $\mathrm{P}_{1} \mathrm{C}_{\mathrm{i}}=0$ & & $\begin{array}{l}\text { MAP } \\
\text { (MAP1 } \\
\text { and } \\
\text { MAP2)- } \\
\text { Managed } \\
\text { Access } \\
\text { Phase } \\
\end{array}$ \\
\hline
\end{tabular}

The following Fig. 2 presents the algorithm that depicts the role of the coordinator node to identify different types of data, e.g. emergency, on-demand or normal. If any data come from body sensor node which is categorized as an emergency and if there is a corresponding level of criticality or emergency type already stored before in the criticality index table, then the status of that particular data traffic will be designated and counted as critical. In our research, we further define and assess the level of criticality of data, as presented in Fig. 3.

a) If the priority-criticality index value $\mathrm{P}_{1} \mathrm{C}_{\mathrm{i}}=\mathrm{P}_{1} \mathrm{C}_{1}$, then the traffic is nominated as extremely very high critical traffic.

$b$ ) If the priority-criticality index value $\mathrm{P}_{1} \mathrm{C}_{\mathrm{i}}=\mathrm{P}_{1} \mathrm{C}_{2}$, then the traffic is nominated as extremely high critical traffic.

c) If the priority-criticality index value $\mathrm{P}_{1} \mathrm{C}_{\mathrm{i}}=\mathrm{P}_{1} \mathrm{C}_{3}$, then the traffic is nominated as extremely critical traffic.

d) If the priority-criticality index value $\mathrm{P}_{1} \mathrm{C}_{\mathrm{i}}=\mathrm{P}_{1} \mathrm{C}_{4}$, then the traffic is nominated as very high critical traffic.

e) And if the priority-criticality index value $\mathrm{P}_{1} \mathrm{C}_{\mathrm{i}}=\mathrm{P}_{1} \mathrm{C}_{5}$, then the traffic is nominated as high critical traffic.

f) If the priority-criticality index value $\mathrm{P}_{1} \mathrm{C}_{\mathrm{i}}=\mathrm{P}_{1} \mathrm{C}_{6}$, then the traffic is nominated as extremely critical traffic.

$g$ ) If the priority-criticality index value $\mathrm{P}_{1} \mathrm{C}_{\mathrm{i}}=\mathrm{P}_{1} \mathrm{C}_{7}$, then the traffic is nominated as moderately critical traffic.

h) If the priority-criticality index value $\mathrm{P}_{1} \mathrm{C}_{\mathrm{i}}=\mathrm{P}_{1} \mathrm{C}_{8}$, then the traffic is nominated as moderately low critical traffic.

i) And if the priority-criticality index value $\mathrm{P}_{1} \mathrm{C}_{\mathrm{i}}=\mathrm{P}_{1} \mathrm{C}_{9}$, then the traffic is nominated as moderately very low critical traffic. $j$ ) If the priority-criticality index value $\mathrm{P}_{1} \mathrm{C}_{\mathrm{i}}=\mathrm{P}_{1} \mathrm{C}_{10}$ then the traffic is nominated as low critical traffic.

k) And if the priority-criticality index value $\mathrm{P}_{1} \mathrm{C}_{\mathrm{i}}=\mathrm{P}_{1} \mathrm{C}_{11}$, then the traffic is nominated as very low critical traffic.

l) If the priority-criticality index value $\mathrm{P}_{1} \mathrm{C}_{\mathrm{i}}=\mathrm{P}_{1} \mathrm{C}_{12}$, then the traffic is nominated as extremely low critical traffic.

Pseudo code 1: To define heterogeneous physiological data types at coordinator level

Input:

Threshold value for each physiological heterogeneous data/packets/traffic (vital sign): threshold value

Physiological data/packets/traffic are sensed by various sensors: data

Output:

Data types' classification by sensor nodes Begin

//Sensors sense data from Pilgrims/Patients and send to coordinator/gateway/hub/sink/master node for further processing

1. if data exceeds ( $>$ or $<$ ) threshold value then Category of data is in emergency condition

Set the highest priority

Send data from coordinator to channel as soon as channel is idle for allocation of slots in superframe for data transmission using

CSMA/CA access mechanism

2. elseif data exceeds $(>$ or $<)$ or within $(<>)$ threshold value

(is sensed due to the request received from healthcare station via the body coordinator) then

Category of data is in on-demand scenario

Set the second highest priority

Coordinator send data received from sensor to channel for allocation of slots for data transmission using CSMA/CA access mechanism

3. elseif data within $(<>)$ threshold value then

Category of data is in normal scenario

Set low priority

Send data from coordinator to channel in allocated time for allocation of slots for data transmission using scheduled access mechanism

\section{4. else \\ Repeat step 1}

\section{5. end if}

\section{End}

Fig. 2. The Role of the Coordinator to Identify Heterogeneous Traffic. 
Pseudo code 2: To handle emergency and non-emergency events along

with the criticality levels of emergency data at coordinator node Input:

T: Traffic that justifies patients'/pilgrims' health status

data type

$\mathrm{P}_{1} \mathrm{C}_{\mathrm{i}}$ : Priority-criticality (severity) index value

Output:

Emergency events classification based on data criticality level

Begin

// for every arrive data from the sensor node

1. if (data type $==$ emergency) $\& \&\left(P_{1} C_{i}==P_{1} C_{1}\right)$ then

$\mathrm{T}$ nominates as extremely very high critical traffic

2. elseif (data type $==$ emergency) $\& \&\left(P_{1} C_{i}==P_{1} C_{2}\right)$ then

$\mathrm{T}$ nominates as extremely high critical traffic

3. elseif (data type $==$ emergency) \& \& $\left(\mathrm{P}_{1} \mathrm{C}_{\mathrm{i}}==\mathrm{P}_{1} \mathrm{C}_{3}\right)$ then

$\mathrm{T}$ nominates as extremely critical traffic

4. elseif (data type $==$ emergency) \&\& $\left(\mathrm{P}_{1} \mathrm{C}_{\mathrm{i}}==\mathrm{P}_{1} \mathrm{C}_{4}\right)$ then

$\mathrm{T}$ nominates as very high critical traffic

5. elseif (data type $==$ emergency) \&\& $\left(P_{1} C_{i}==P_{1} C_{5}\right)$ then

T nominates as high critical traffic

6. elseif (data type $==$ emergency) $\& \&\left(\mathrm{P}_{1} \mathrm{C}_{\mathrm{i}}==\mathrm{P}_{1} \mathrm{C}_{6}\right)$ then

$\mathrm{T}$ nominates as critical traffic

7. elseif (data type $==$ emergency) \&\& $\left(\mathrm{P}_{1} \mathrm{C}_{\mathrm{i}}==\mathrm{P}_{1} \mathrm{C}_{7}\right)$ then

$\mathrm{T}$ nominates as moderately critical traffic

8. elseif (data type $==$ emergency) \& \& $\left(\mathrm{P}_{1} \mathrm{C}_{\mathrm{i}}==\mathrm{P}_{1} \mathrm{C}_{8}\right)$ then

$T$ nominates as moderately low critical traffic

9. elseif (data type $==$ emergency) $\& \&\left(P_{1} C_{i}==P_{1} C_{9}\right)$ then

$T$ nominates as moderately very low critical traffic

10. elseif (data type $==$ emergency) $\& \&\left(P_{1} C_{i}==P_{1} C_{10}\right)$ then

$T$ nominates as low critical traffic

11. elseif (data type $==$ emergency) $\& \&\left(P_{1} C_{i}==P_{1} C_{11}\right)$ then

$T$ nominates as very low critical traffic

12. elseif (data type $==$ emergency) $\& \&\left(P_{1} C_{i}==P_{1} C_{12}\right)$ then

$\mathrm{T}$ nominates as extremely low critical traffic

13. else

if $($ data type $==$ on-demand $) \& \&\left(\mathrm{P}_{1} \mathrm{C}_{\mathrm{i}}==0\right)$ then

$\mathrm{T}$ nominates as on-demand

14. end if

15. else

16. if (data type $==$ no event or normal $) \& \&\left(P_{1} C_{i}==0\right)$ then

$T$ nominates as normal traffic

17. end if

18. end if

19. end

Fig. 3. The Role of the Coordinator to Deal with Emergency Traffic.

The following Fig. 4 shows the deployment of CSMA/CA mechanism for the proposed model and for that the user priority is set to $\mathrm{UP}_{\mathrm{i}}$ where, $\mathrm{i}=5-7$ for normal, on-demand and emergency traffic respectively. The proposed WBAN should handle heterogeneous traffic hence for the transmission of emergency traffic the length of back off counter (BC) must be minimal, otherwise for normal medical data the length of $\mathrm{BC}$ can be more. And data is transmitted when the $\mathrm{BC}=0$. Moreover, in this emergency data transmission case the contention window $(\mathrm{CW})$ is set to a minimum as $\mathrm{CW}=\mathrm{CW}_{\text {min }}$. for the rest of the cases, the $\mathrm{CW}$ is set to $\mathrm{CW}_{\text {max. In the case of }}$ transmission of the normal medical data, the coordinator needs to wait until the emergency data are all transmitted.

\section{Analytical Model of the Proposed Priority MAC Protocol M/M/1 Queue based Preemptive Method}

In this research, the overall delay is calculated as the addition of transmission delay and the queue delay. Average data transmission time is considered as the packet transmission delay from the source to the destination node. The queue delay demonstrates the moment when the packet departs or leaves the queue due to not successfully transmission of packets. The service time is being served as the queue delay in our queuing model. We consider the average delay to analysis the performance of our analytical model. The delay can be calculated for extremely very high critical traffic, extremely high critical traffic, extremely critical traffic, very high critical traffic, and high critical traffic as shown in quations (1), (2), (3), (4), and (5), respectively.

$$
\begin{aligned}
& \mathrm{E}\left(t_{\mathrm{p} 1 \mathrm{c} 1}\right)=\frac{\frac{1}{\mu}}{1-\rho_{\mathrm{p} 1 \mathrm{c} 1}} \\
& \mathrm{E}\left(t_{\mathrm{p} 1 \mathrm{c} 2}\right)=\frac{\mathrm{E}\left(\mathrm{n}_{\mathrm{p} 1 \mathrm{c} 2}\right)}{\lambda \mathrm{p} 1 \mathrm{c} 2}=\frac{\frac{1}{\mu}}{\left(1-\rho_{\mathrm{p} 1 \mathrm{c} 1}\right)\left(1-\rho_{\mathrm{p} 1 \mathrm{c} 1}-\rho_{\mathrm{p} 1 \mathrm{c} 2}\right)} \\
& \mathrm{E}\left(t_{\mathrm{p} 1 \mathrm{c} 3}\right)=\frac{\mathrm{E}\left(\mathrm{n}_{\mathrm{p} 1 \mathrm{c} 3}\right)}{\lambda \mathrm{p} 1 \mathrm{c} 3}=\frac{\frac{1}{\mu}}{\left(1-\rho_{\mathrm{p} 1 \mathrm{c} 1}-\rho_{\mathrm{p} 1 \mathrm{c} 2}\right)\left(1-\rho_{\mathrm{p} 1 \mathrm{c} 1}-\rho_{\mathrm{p} 1 \mathrm{c} 2}-\rho_{\mathrm{p} 1 \mathrm{c} 3}\right)} \\
& \mathrm{E}\left(t_{\mathrm{p} 1 \mathrm{c} 4}\right)=\frac{\mathrm{E}\left(\mathrm{n}_{\mathrm{p} 1 \mathrm{c} 4}\right)}{\lambda \mathrm{p} 1 \mathrm{c} 4}=\frac{\frac{1}{\mu}}{\left(1-\rho_{\mathrm{p} 1 \mathrm{c} 1}-\rho_{\mathrm{p} 1 \mathrm{c} 2}-\rho_{\mathrm{p} 1 \mathrm{c} 3}\right)\left(1-\rho_{\mathrm{p} 1 \mathrm{c} 1}-\rho_{\mathrm{p} 1 \mathrm{c} 2}-\rho_{\mathrm{p} 1 \mathrm{c} 3}-\rho_{\mathrm{p} 1 \mathrm{c} 4}\right)} \\
& \mathrm{E}\left(t_{\mathrm{p} 1 \mathrm{c} 5}\right)=\frac{\mathrm{E}\left(\mathrm{n}_{\mathrm{p} 1 \mathrm{c} 5}\right)}{\lambda \mathrm{p} 1 \mathrm{c5}}=\frac{\frac{1}{\mu}}{\left(1-\rho_{\mathrm{p} 1 \mathrm{c} 1}-\rho_{\mathrm{p} 1 \mathrm{c} 2}-\rho_{\mathrm{p} 1 \mathrm{c} 3}-\rho_{\mathrm{p} 1 \mathrm{c} 4}\right)\left(1-\rho_{\mathrm{p} 1 \mathrm{c} 1}-\rho_{\mathrm{p} 1 \mathrm{c} 2}-\rho_{\mathrm{p} 1 \mathrm{c} 3}-\rho_{\mathrm{p} 1 \mathrm{c} 4}-\rho_{\mathrm{p} 1 \mathrm{c} 5}\right)}
\end{aligned}
$$

For $\mathrm{n}$ different severity level of traffic, the delay can be formulated as follows:

$$
\begin{aligned}
& \mathrm{E}\left(n_{\mathrm{p} 1 \mathrm{i}}\right)= \\
& \frac{\rho_{p 1 c 1}+\rho_{p 1 c 2}+\rho_{p 1 c 3}+\rho_{p 1 c 4}+\rho_{p 1 c 5}}{1-\rho_{p 1 c 1}-\rho_{p 1 c 2}-\rho_{p 1 c 3}-\rho_{p 1 c 4}-\rho_{p 1 c 5}}-\frac{\rho_{p 1 c 1}+\rho_{p 1 c 2}+\rho_{p 1 c 3}+\rho_{p 1 c 5}}{1-\rho_{p 1 c 1}-\rho_{p 1 c 2}-\rho_{p 1 c 3}-\rho_{p 1 c 4}} \\
& =\frac{\rho_{p 1 c i}}{\left(1-\left(\sum_{i=1}^{n-1} \rho_{p 1 c_{i}}\right)\right)\left(1-\left(\sum_{i=1}^{n} \rho_{p 1 c_{i}}\right)\right)}
\end{aligned}
$$

And by applying Little's law we get,

$$
\mathrm{E}\left(t_{\mathrm{p} 1 \mathrm{ci}}\right)=\frac{E\left(n_{p 1 c i}\right)}{\lambda p 1 c i}=\frac{\frac{1}{\mu}}{\left(1-\left(\sum_{i=1}^{n-1} \rho_{p 1 c_{i}}\right)\right)\left(1-\left(\sum_{i=1}^{n} \rho_{p 1 c_{i}}\right)\right)}
$$

Over a communication channel, at the destination, throughput is measured as the number of packets correctly received at the destination point in a unit time (the average transmission rate). Kbps is considered as the unit of throughput.

Throughput measures the actual level of network traffic between the transmitting node and receiving nodes. The throughput is calculated as follows.

Throughput $=\frac{\Sigma \text { Packets received }}{\Sigma \text { Packets generated }}$ 


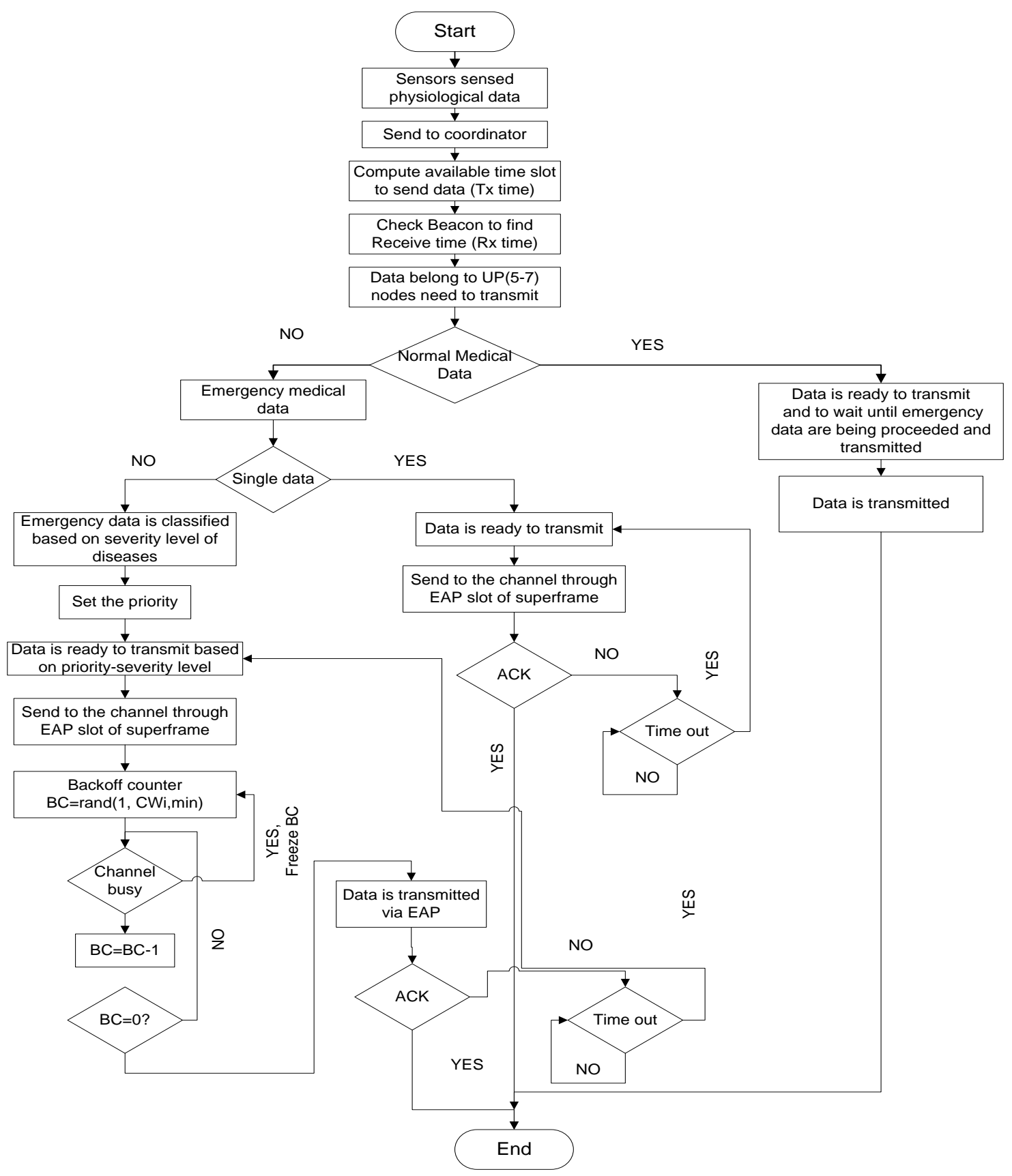

Fig. 4. Operational Flowchart of the Proposed MAC Protocol based on CSMA/CA Mechanism.

Efficient and the low energy consumption is considered as the fundamental requirement of designing MAC protocol for WBAN and its associated networks. The behaviour of wireless network nodes depends on energy consumption and the way to manage it. A network having low traffic consumes less energy than the one with heavy traffic. In this research, the energy consumption of the network is calculated as the cumulative sum of energy consumed by all the sensor nodes. Where energy consumed by a single node is defined as how much remaining energy is subtracting from the initial (preliminary) energy. To systematically and comprehensively evaluate the MAC protocols of WBAN applications, we evaluate energy efficiency by calculating average energy consumption per bit.
At the time of a network simulation run, the energy consumption per bit is calculated as the ratio of the total energy (power) consumption over the total number of bits delivered. Hence, the total energy consumed can be computed as follows.

$$
\begin{aligned}
& \mathrm{E}_{\text {tec }}=\mathrm{T}_{\text {wakeup }} \times \mathrm{P}_{\text {wakeup }}+\mathrm{T}_{\mathrm{tx}} \mathrm{x} \mathrm{P}_{\mathrm{tx}}+\mathrm{T}_{\mathrm{rx}} \times \mathrm{P}_{\mathrm{rx}}+ \\
& \mathrm{T}_{\text {sleep }} \times \mathrm{P}_{\text {sleep }}
\end{aligned}
$$

The total dropped packets are considered as the difference between total transmitted packets (data and control) and total received packets (data \& control) during the data transmission session. Thus, packet drop ratio at the destination node is calculated and defined as the total number of dropped packets 
over total transmitted packets. The the packets dropped loss ratio can be formulated as follows.

Packets loss ratio $=\frac{\text { Number of lost packets }}{\text { Number of received packets }}$

\section{E. Simulation Environment}

The proposed protocol is simulated using Castalia simulator. In this simulation, sensors generate heterogeneous medical packets in term of priority and sensitivity.

\section{F. Simulation Parameters}

To observe the performance of the proposed priority MAC protocol, various scenarios are considered to implement and evaluate include variation in a number of nodes (network size), and traffic sizes up. Varied network sizes ranging from 1 node to 12 nodes are considered to evaluate the performance of the protocol for different sized network. On the other hand, various traffic sizes ranging from 16 bytes to 127 bytes are supposed to assess the performance of the protocol. Correlated with other simulation environments, these nodes are considered with a transmission range of 5 to 10 meters. The operating frequency is set to be $2.4 \mathrm{GHz}$ ISM band, and the bandwidth of the channel is considered to be $250 \mathrm{kbps}$. The simulation parameters are summarized in Table III.

TABLE III. SIMULATION PARAMETERS

\begin{tabular}{|l|l|}
\hline Parameters & Value \\
\hline Number of nodes & 12 \\
\hline Nodes transmission range & From sensing range to max $5 \mathrm{~m}$ to $10 \mathrm{~m}$ \\
\hline mMaxBANSize & $<64$ nodes \\
\hline MAC & IEEE 802.15 .6 \\
\hline Channel mode & Wireless Model \\
\hline Superframe size & 255 slots \\
\hline Superframe Duration & $122.88 \mathrm{~ms}$ \\
\hline Simulation time or runtime & 150 Seconds \\
\hline $\begin{array}{l}\text { Frequency band/ Operating } \\
\text { Frequency }\end{array}$ & $2.4 \mathrm{GHz}$ ISM \\
\hline $\begin{array}{l}\text { Channel or Data rates or } \\
\text { Bandwidth }\end{array}$ & Various data rates up to $250 \mathrm{kbps}$ \\
\hline Area Size & $5 \mathrm{~m}$ x $5 \mathrm{~m}$ \\
\hline Packet Size & Variable, up to 512 bytes \\
\hline macMaxCSMABackoffs & $*$ So far there is no specific unit \\
\hline Initial energy & 1 Joule \\
\hline Transmission power consumption & $12.3 \mathrm{~mA}$ \\
\hline Reception power consumption & $14 \mathrm{~mA}$ \\
\hline Idle power consumption & $0.4 \mathrm{~mA}$ \\
\hline Beacon size & $40 \quad$ ytes \\
\hline
\end{tabular}

\section{RESULT AND DISCUSSION}

\section{A. Performance Evaluation and Result Discussion}

The performance of the priority MAC protocol is analyzed and evaluated for diverse scenarios, and QoS conditions such as delay, energy, throughput, and packets dropped rate considering the variation of sensor nodes in the network and different packet sizes up. We assume a star topology WBAN with a single hop and heterogeneous traffic or data. In our proposed analytical model for the MAC superframe structure, the length of EAP2, RAP2 and CAP is set to zero. We assume that data can access the channel without RTS/CTS mechanism, and data retransmission may occur if there is any collision. The performance of the MAC model is compared with TA-MAC protocol and TP-CAT protocol, and the simulation results are depicted in the subsequent sub-sections.

\section{B. First Scenario}

In the first scenario, delay and energy consumption comparison for urgent and normal traffic under varying nodes for the Proposed Priority MAC Protocol is portrayed.

Fig. 5 illustrates the situation where the network size (the number of nodes) increases for both emergency and normal medical cases from 1 to 12 with the constant packets size 16 Bytes. It has been found that the delay for both normal and emergency medical traffic increases with the increasing number of nodes.

Hence, the increase in overall delay is due to the increased number of nodes and packets generated, thus increased wait, collisions and retransmissions in the network. In the implementation of the proposed priority MAC protocol, we assume there is no data collision and retransmission in the network since we deploy a preemptive priority queue scheme and the modified MAC superframe structure.

Therefore, in the proposed priority MAC protocol deploying technique, the emergency medical traffic proceeds ahead of normal medical traffic and to access the channel faster than normal medical traffic. Hence the delay in emergency traffic is much less than normal medical traffic.

The energy consumption rate for normal medical traffic is higher than that of emergency medical traffic because normal traffic needs to wait more in the network for being transmitted to the communication channel than emergency traffic. If the delay increases in the network drastically for the normal traffic, then there is a more possibility of more energy consumption whereas the level of energy consumption is lower for emergency traffic as presented in Fig. 6.

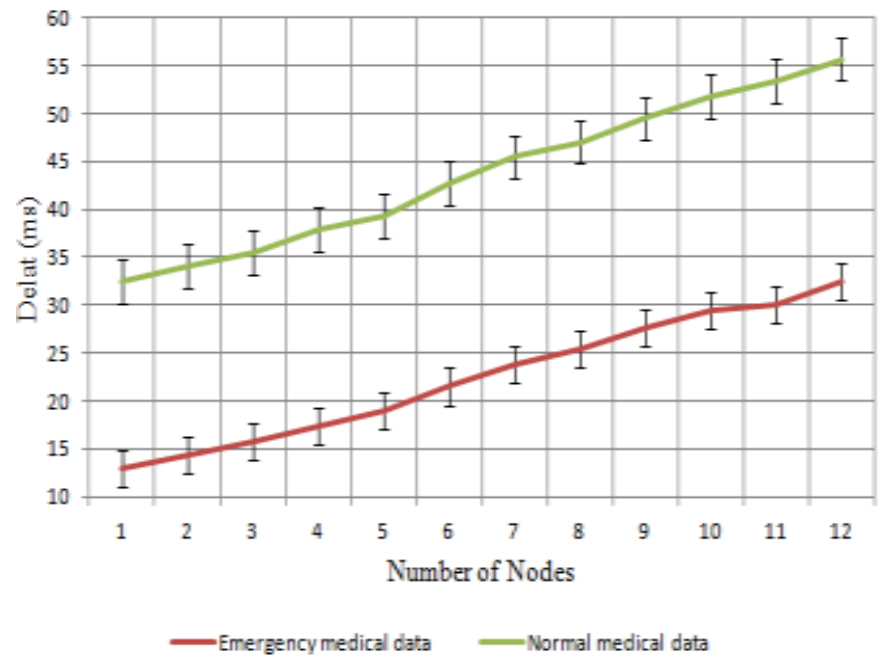

Fig. 5. Delay Assessment for Emergency Medical and Normal Medical Traffics under Varying Sensor Nodes in the Network. 


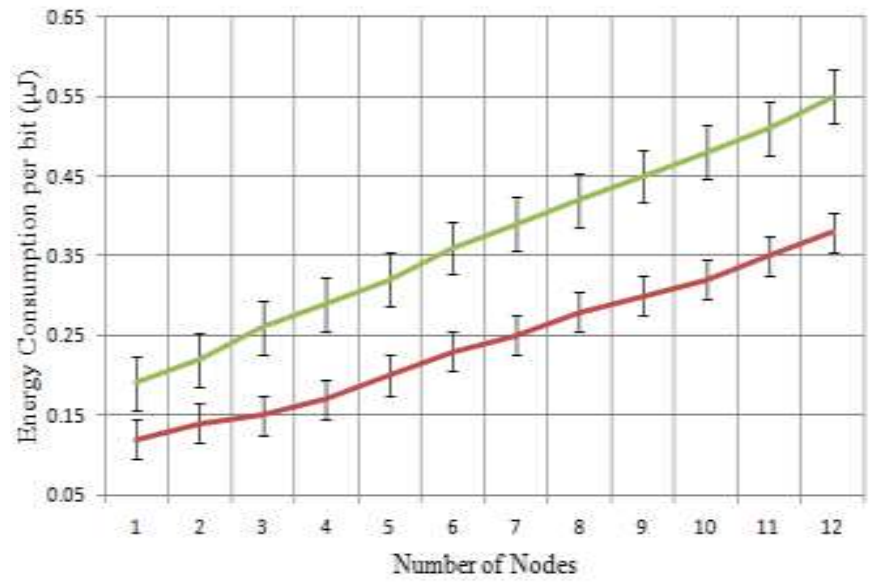

Emergencymedical dato Normal medical data

Fig. 6. Energy Consumption Assessment for Emergency Medical and Normal Medical Traffics under Varying Sensor Nodes in the Network.

\section{Second Scenario}

In the second scenario, the performance analysis of different emergency traffic considering their level of priorities for the proposed priority MAC protocol is presented.

In this section, the performance of different emergency traffic is analyzed based on their priorities and level of criticality. The results are determined considering variation in traffic sizes.

It is observed that at $2.4 \mathrm{GHz}$ frequency band and 250 kbps data rate, the emergency traffic with the highest priority can send data faster than that of lower priority. The results differ with the number of packets sizes up to 127 bytes.

Here, user priority seven is considered for emergency traffic. However, the difference in delay values between $\mathrm{P}_{1} \mathrm{C}_{1}$ (Extremely very high critical data) extremely very high critical emergency traffic and $\mathrm{P}_{1} \mathrm{C}_{12}$ (Extremely Low critical data) extremely low critical traffic under WBAN user priority seven is observed. $\mathrm{P}_{1} \mathrm{C}_{1}$ extremely very high critical emergency traffic provides less delay than other low critical emergency traffic because $\mathrm{P}_{1} \mathrm{C}_{1}$ (Extremely very high critical data) gets earlier and more channel opportunities. However, the cumulative delay of 12 emergency data and normal data is equal to the average delay of twelve nodes during the medical normal and emergency events as presented earlier in Fig. 5. The data collision and data retransmission possibility are less since we deploy preemptive priority queue at the MAC level that ensures fast and low delay data communication which is illustrated in Fig. 7.

Also, emergency traffic with the highest critical index $\mathrm{P}_{1} \mathrm{C}_{1}$ (Extremely very high critical data) consumes less energy than emergency traffic with lower criticality index. Include $\mathrm{P}_{1} \mathrm{C}_{2}$ (Extremely high critical data), $\mathrm{P}_{1} \mathrm{C}_{3}$ (Extremely critical data), $\mathrm{P}_{1} \mathrm{C}_{4}$ (Very high critical data), $\mathrm{P}_{1} \mathrm{C}_{5}$ (High critical data) and so forth until $\mathrm{P}_{1} \mathrm{C}_{12}$ (Extremely low critical data). However, the energy consumption increases for all traffic classes according to packet size up, and the result is illustrated in Fig. 8.

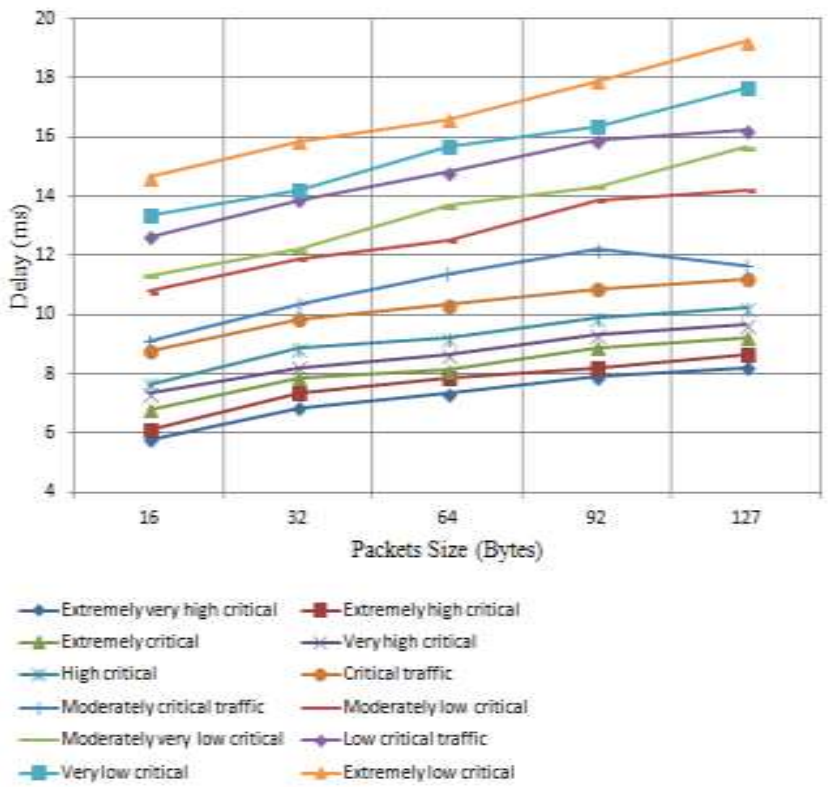

Fig. 7. Average Delay (ms) of Emergency Traffic is Analyzed based on their Priorities and Criticality Level as Compared to Packet Sizes up.

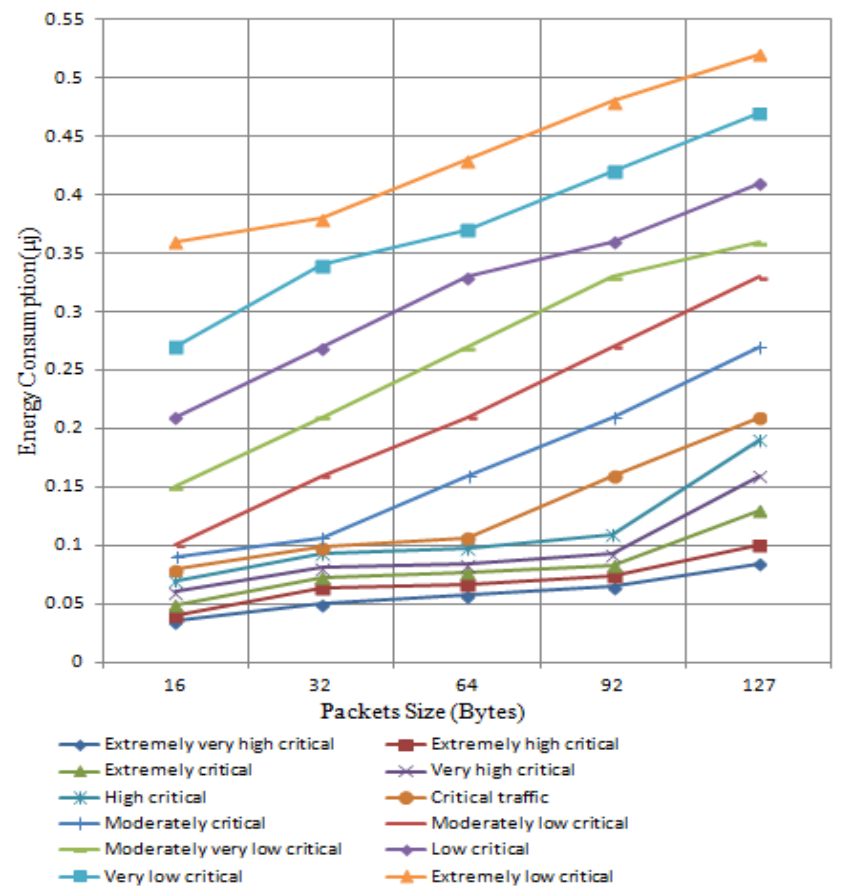

Fig. 8. Overall Energy (Power) Consumption Per Bit $(\mu \mathrm{j})$ of Emergency Traffic is Analyzed based on their Priorities Level as Compared to Packet Sizes up.

\section{Third Scenario}

In the third scenario, the performance analysis and evaluation of proposed priority MAC protocol based on heterogeneous traffic, considering variation in the number of sensor nodes (Network Size) are discussed.

For all the distinct circumstances, we assign the maximum number of sensor nodes as 12 because under 12, the proposed IEEE 802.15.6 priority MAC protocol and other competitive 
MAC protocols such as TA-MAC protocol and TP-CAT protocol show performance inconsistency and difference. It is remarkable that for a maximum number of sensor nodes 12 , the average delay of the proposed MAC sharply decreases, which is less than TA-MAC protocol and TP-CAT protocol, as shown in Fig. 9.

In a wireless network, an increase in the number of nodes may increase the number of traffic load; hence, collisions, data retransmissions also increase. However, in the proposed priority MAC protocol, the CAP is set to zero. And the EAP phase is fixed for emergency data access, hence, contention in our proposed priority MAC is significantly less than that of the TP-CAT protocol and TA MAC protocol, resulting in a lower number of collisions, and therefore lower energy consumption. Fig. 10 portrays the average power consumption per bit basis.

Fig. 11 shows the overall network throughput of the priority MAC protocol, TA-MAC protocol and TP-CAT protocol. Here, in this research, when the network has increased number of sensor nodes (in our case nodes range 112) or traffic load, then the throughput of all three protocols such as priority MAC, TP-CAT protocol and TA-MAC protocol also increases. Our proposed priority MAC protocol shows enhanced throughput over TA-MAC and TP-CAT protocol with the increased number of sensor nodes. Data classification and prioritization mechanism, EAP allocation for emergency data transmission make the priority MAC protocol of this research outperforms TA-MAC and TP-CAT protocol.

In a wireless network, an increase in the number of nodes may increase the amount of traffic load; hence, collisions, data retransmissions also may increase thus results in raising the percentage of packets dropped rate. However, as discussed in earlier sections, in the proposed priority MAC protocol, the CAP is set to zero, and the EAP phase is fixed for emergency data access. Hence, contention and packets retransmission in the proposed priority MAC is significantly less than that of TP-CAT and TA-MAC protocol, resulting in a lower number of collisions. Therefore lower packets dropped rate in our proposed priority MAC protocol. The packet dropped rate is shown in Fig. 12.

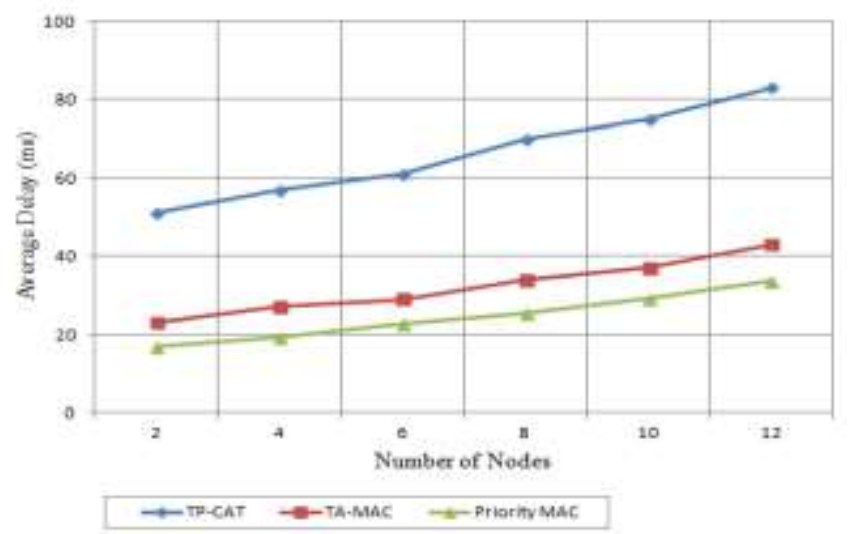

Fig. 9. Average Delay in Millisecond (ms) as Compared to the Number of Nodes.

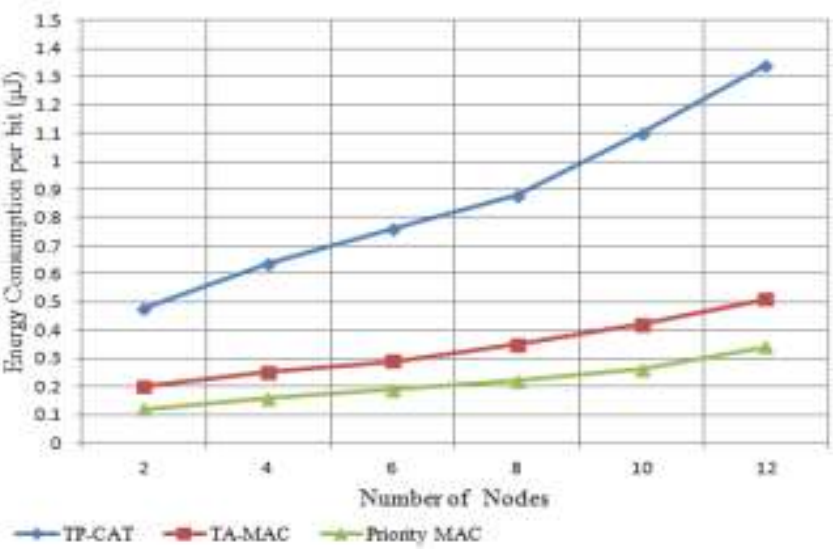

Fig. 10. Average Power (Energy) Consumption Per Bit.

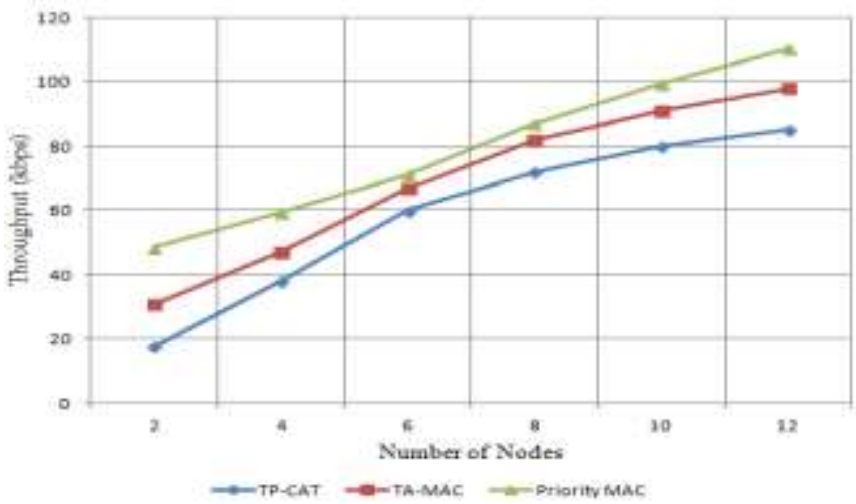

Fig. 11. Overall Network Throughput.

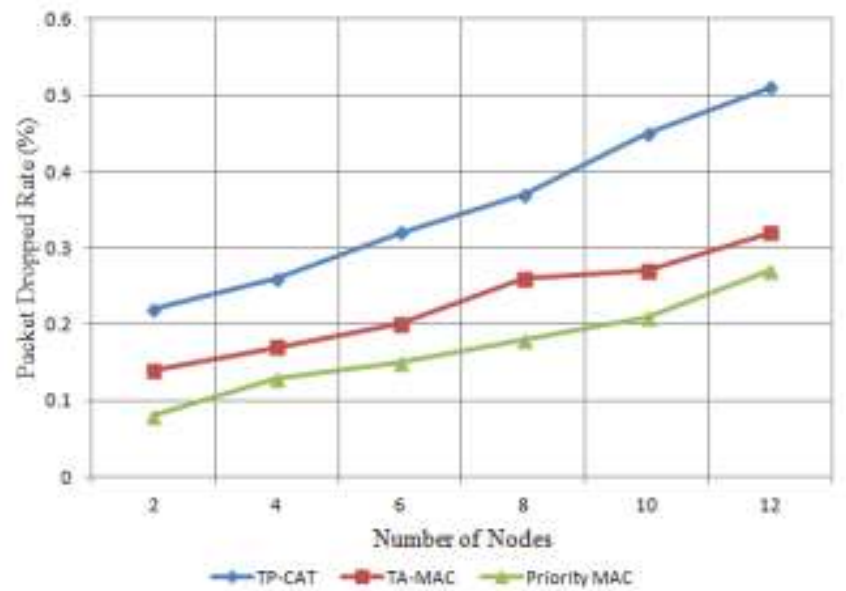

Fig. 12. Packets Dropped Rate (\%).

\section{CONCLUSION}

To monitor pilgrims' health in a real-time manner, several issues are considered in designing an $\mathrm{M} / \mathrm{M} / 1$ preemptive queue-based priority MAC protocol. First, pilgrims emergencies are categorized and prioritized based on the level of criticality. Second, algorithms are developed to define the role of different sensors and the coordinator. Third, the MAC superframe structure is improved according to the IEEE 802.15.6 standard for fast and collision-free data transmission. 
Fourth, to reduce the delay of physiological data transmission, an $\mathrm{M} / \mathrm{M} / 1$ preemptive priority queue model is proposed and minimal backoff period is considered. Fifth, to improve energy efficiency, sleep and the wake-up mechanism is used. The proposed priority MAC protocol has been simulated using the Castalia simulator. Results are compared with the most recent traffic adaptive based MAC protocol (TA-MAC) and traffic priority based channel access technique (TP-CAT) protocol. Results demonstrate that delay, data loss rate, and the average energy consumption are relatively low. The throughput is high during emergency data transmission in a network due to no data retransmission and collision. Our future plan is to experiment the proposed MAC protocol with non-preemptive priority queue method. And also for broader types of biomedical data with boundless QoS issues.

\section{ACKNOWLEDGMENT}

We would like to express our gratitude to IIC University of Technology, Cambodia and Najran University, Saudi Arabia for providing us excellent research environment.

\section{REFERENCES}

[1] WHO report on Hajj: http://www.who.int/csr/ITH_final.pdf.

[2] MoH KSA guideline on Hajj: https://www.moh.gov.sa/en/Hajj1432/ Awareness/Documents/DlelHaj-E.pdf.

[3] Abdullah, A., Jamil, A., \& Memish, Z. (2012). Patternofdiseasesamon gvisitorstoMinahealthcentersduringtheHajjseason, 1429H(2008G). JournalofInfectionandPublicHealth, 5, 22-34.

[4] Ahmed, Q. A., Arabi, Y. M., \& Memish, Z. A. (2006). Health risks at the Hajj. Lancet, 367(9515), 1008-1015. https://doi.org/10.1016/S01406736(06)68429-8.

[5] Ahmed, Q. A., Arabi, Y. M., \& Memish, Z. A. (2015). Health risk at the Hajj. 6736(APRIL 2006). https://doi.org/10.1016/S01406736(06)68429-8

[6] Alkar ,A.Z and Karaca,M.A,2009, "An Internet Based Interactive Embedded Data-Acquisition System for Real-Time Applications", IEEE Transactions on Instrumentation and Measurement,Vol.58,No.3.pp. 522.

[7] AladdeinAmro, Qasem Abel-MutiNijem, 2012Pilgrims "Hajj" Tracking System (emutawiff)," Contemporary Engineering Sciences, Vol.5,No.9,p.p.437-446.

[8] Jaafar, A.D. ; Aris, M.F.M. ; Ayu, M.A., HajjLocator: A Hajj pilgrimage tracking framework in crowded ubiquitous environment, International Conference on Multimedia Computing and Systems (ICMCS), 2011, page 1-6, Print ISBN: 978-1-61284-730-6.
[9] Lionel M.Ni and Dian Zhang ,Michael.R.Souryal,2011, "RFID-Based Localization and Tracking Technologies",IEEEWireless Communication. [8]Teddy Mantoro,Media.A.Ayu,Murni Mahmud,2012, "HajjCrowd Tracking in a PersiveEnvironment",International Journal of Mobile Computing and Multimedia Communication,4(2),11-2.

[10] O. Tayan, 'A Proposed Model for Optimizing the Flow of Pilgrims between Holy Sites During Hajj Using Traffic Congestion Control', Proceedings of the International Journal of Engineering and Technology, Publication Date: April 10, 2010.

[11] L. J. Akinbami, J. E. Moorman, and X. Liu, "Asthma prevalence, health care use, and mortality: United States, 2005-2009.," National health statistics reports, no. 32, pp. 1-14, Jan. 2011.

[12] Yuan, D., Zheng, G., Ma, H., Shang, J., \& Li, J. (2019). An Adaptive MAC Protocol Based on IEEE802.15.6 for Wireless Body Area Networks. Wireless Communications and Mobile Computing, 2019, 19. https://doi.org/10.1155/2019/3681631.

[13] Rismanian Yazdi, F., Hosseinzadeh, M., \& Jabbehdari, S. (2019). A Priority-Based MAC Protocol for Energy Consumption and Delay Guaranteed in Wireless Body Area Networks. Wireless Personal Communications, (May). https://doi.org/10.1007/s11277-019-06490-z.

[14] Gouda, K. C., Biswal, S. P., Debnath, S., Sahu, S. K., Science, C., Online, F., \& Notes, L. (2019). Implementation of Traffic Priority Aware Medium Access Control Protocol for Wireless Body Area Networks. Springer Link, 1-7.

[15] Bhandari, S., \& Moh, S. (2019). A Mac Protocol with Dynamic Allocation of Time Slots Based on Traffic Priority in Wireless Body Area Networks. International Journal of Computer Networks \& Communications, 11(4), 25-41. https://doi.org/10.5121/ijcnc.2019.11402.

[16] Ambigavathi, M., \& Sridharan, D. (2018c). Traffic Priority Based Channel Assignment Technique for Critical Data Transmission in Wireless Body Area Network. Journal of Medical Systems, 42(11). https://doi.org/10.1007/s10916-018-1054-y.

[17] Henna, S., Sajeel, M., Bashir, F., Asfand-E-Yar, M., \& Tauqir, M. (2017). A fair contention access scheme for low-priority traffic in wireless body area networks. Sensors (Switzerland), 17(9). https://doi.org/10.3390/s17091931.

[18] Ullah, F., Abdullah, A. H., Kaiwartya, O., Lloret, J., \& Arshad, M. M. (2017). EETP-MAC: energy efficient traffic prioritization for medium access control in wireless body area networks. Telecommunication Systems, 1-23. https://doi.org/10.1007/s11235-017-0349-5.

[19] Gündoğdu, K., \& Çalhan, A. (2016). An Implementation ofWireless Body Area Networks for Improving Priority Data Transmission Delay. Journal of Medical Systems, 40(75).

[20] Castalia simulator: http://castalia.npc.nicta.com.au. 